\title{
FiWiN5G - Fiber-Wireless Integrated Networks for 5th Generation delivery
}

\author{
John E. Mitchell, Senior Member, IEEE \\ Department of Electronic and Electrical Engineering, University College London, London, WC1E 7JE, UK \\ Tel: +442076793281,e-mail:j.mitchell@ucl.ac.uk,Twitter: @drjohnmitchell
}

\begin{abstract}
This paper describes the work and structure of the FIWIN5G: FIber-Wireless Integrated Networks for 5th Generation delivery, an Innovative Training Network (ITN) funded by the European Union's Horizon 2020 research and innovation programme through the Marie Sklodowska-Curie Actions (MSCA) scheme. The programme, which started in January 2015, comprises 10 leading research institutions, a wide range of industrial partners (from multi-nationals to SMEs) and a comprehensive research training program for all 15 Early Stage Researchers (ESR) recruited.
\end{abstract}

Keywords: European Networks, 5G, Photonic Technologies, Microwave Photonics.

\section{INTRODUCTION}

FIWIN5G: FIber-Wireless Integrated Networks for 5th Generation delivery, which started in January 2015 is an ambitious Innovative Training Network (ITN) funded by the European Union's Horizon 2020 research and innovation programme through the Marie Sklodowska-Curie Actions (MCSA) scheme. It comprises 10 leading research institutions, a broad range of supporting associate partners from industry, and a comprehensive research training program for all 15 Early Stage Researchers (ESR) who have been recruited to the network over the past year. The 10 reseach partners of the project cover 9 EU member states; University College London (UCL) - UK, University of Duisburg-Essen (UDE) - Germany, Technical Unviersity of Denmark (DTU) - Denmark, Grenoble Institute of Technology (GINP) - France, Universidad Carlos III de Madrid (UC3M) - Spain, University of Cyprus - Cyprus, University of Ljubljana - Slovenia, Budapest University of Technology and Economics (BME) - Hungary, National Interuniversity Consortium for Telecommunications (CNIT) - Italy and III-V Lab - France. Industrial partner organisations include, Thales, BAE systems, InLambda, Finisar, Rohde \& Schwarz, TEEM Photonics, Ericsson Telecomunicazioni SPA, Ericsson R\&D HU, Siklu and DAS Photonics.

FiWiN5G aims to produce the next generation of researchers who will enable Europe to take a leading role in the multidisciplinary area of $5 \mathrm{G}$ high-speed wireless internet and beyond, involving devices, systems and networks. To achieve this, the network integrates multidisciplinary scientific expertise, complementary skills, and experience working in academia and industry to empower ESRs to work in interdisciplinary teams, integrate their activities, share expertise, and promote a vision of a converged wireless and optical access network that seamlessly and efficiently supports the services and applications being demanded.

The support of high bandwidth and high mobility is central to the driving vision of future networks, requiring a large number of technologies to converge, co-exist and interoperate, and most importantly, cooperate, if this vision is to be efficiently and cost-effectively realised. A key area within this next generation jigsaw is the integration of optical fibre networks and radio networks at mm-wave frequencies, to provide high-bandwidth front/backhaul services and enable scalable and manageable networks without a highly complex interface structure and multiple overlaid protocols.

\section{FOCUS OF THE PROGRAMME}

Broadband wireless access is widely considered as the third wireless revolution, following cellular phones in the 90 s and Wi-Fi in the 2000. It is viewed by many wireless carriers as a disruptive technology. Several solutions have already been proposed for $10 \mathrm{~Gb} / \mathrm{s}$ broadband wireless systems a number of which employ photonic technologies. Today, various companies are offering commercial series broadband wireless link products, typically up to $1.25 \mathrm{~Gb} / \mathrm{s}$, potentially supporting full-duplex $1 \mathrm{~Gb}$ Ethernet. Products are available from Bridgewave Communication, Siklu, EBCC, Comotech, Loea, Elva-1, LightPointe, Gigabeam, HXI and SIAE. Most of those systems operate within the V-band around $60 \mathrm{GHz}$ or the E-band around 70 and $80 \mathrm{GHz}$ and are equipped with optical fiber interfaces. Usually, the spectral efficiency offered by those systems is low (around $1 \mathrm{bit} / \mathrm{sec} / \mathrm{Hz}$ ), and currently, as far as we are award, none of these systems would allow integration into a WDM network; they all require Gigabit Ethernet inputs. Broadband wireless systems offering $10 \mathrm{~Gb} / \mathrm{s}$ connectivity are currently under development or are just being announced, e.g. by Asyrmatos or Gigabeam. Most of those so- 
called super-broadband wireless systems also operate in the V-band and the E-band, although the Asyrmatos product also considers operation in frequencies around $94 \mathrm{GHz}$ and $140 \mathrm{GHz}$.

Looking at the academic achievements and developments, it should be stated that an increasing number of research institutes and groups are currently working to enable broadband wireless $10 \mathrm{~Gb} / \mathrm{s}$ access and beyond. Most of all relevant basic research on broadband mm-wave wireless systems with high capacities are utilizing photonic techniques. Besides the Japanese research group from NTT, US based groups and companies such as Battelle, Corning or Georgia Tech have built a photonic wireless system operating at millimeter-wave frequencies. Radio-over-Fiber (RoF) or millimeter-wave-over-fiber techniques have long been exploited for high-capacity wireless systems [1-9]. Also the European commission has, for a number of years, supported physical layer developments in this domain, e.g. within the IPHOBAC and now the IPHOBAC-NG project [1,2] as well as other projects. Although Radio-over-Fiber is a well-established field of research, we would argue that most of the previous results included only limited demonstrations and were based on immature technologies that lacked full intergration. However, these previous results were essential. The demonstration that wireless links could operate at fiber speeds as, for example, shown in IPHOBAC [3] have opened-up new opportunities, with new business units being founded in this field.

The advantages of using Radio-over-Fiber (RoF) techniques are multifold: it provides a scalable technology that allows seamless integration of the optical access network and the transmitting antenna by providing direct optical baseband to optical RF up-conversion. The low transmission loss of optical fiber allows for antenna remoting, allowing centralizing wireless carrier generation and signal processing. Photonics is generally of advantage when generating and transmitting spectrally-broad and spectrally-efficient ultra-high capacity data signals.

Photonic techniques have become key enablers to unlock future broadband wireless communications with multigigabit data rates in order to support the current trends of mobile data traffic, which is expected to increase 13-fold between 2012 and 2017 [14]. Photonic techniques are an alternative approach having some inherent key advantages such as being broadly tunable, having an ultra-wide bandwidth and being able to be seamlessly connected to wired (fiber optic) networks. The two key components for realizing photonics-based continuouswave $(\mathrm{CW})$ generation of millimeter waves are optical signal sources together with optical-to-electrical $(\mathrm{O} / \mathrm{E})$ converters [7]. FiWiN5G aims to demonstrated significant advances in these two technology areas in combination with state-of-the-art systems demonstrations.

\subsection{Systems demonstrations of Photonic Technologies for 5G networks}

Most systems operate in the $60 \mathrm{GHz}$ band and utilized rather simple modulation formats such as optical On-Off Keying (OOK), [4,5]. Recent UDE [6] demonstrated a $60 \mathrm{GHz}$ millimeter-wave-over-fiber system providing $12.5 \mathrm{~Gb} / \mathrm{s}$ with OOK modulation. The bandwidth and spectral efficiencies for DSB-OOK systems providing 10 $\mathrm{Gb} / \mathrm{s}$ capacities is typically around $16-18 \mathrm{GHz}$ and $0.5-0.7 \mathrm{bit} / \mathrm{s} / \mathrm{Hz}$, respectively. This could be slightly improved by using SSB-OOK but in order to cope with the bandwidth regulations, modulation formats with higher spectral efficiencies have been used. Within the IPHOBAC project UDE and France Telecom - Orange developed a millimeter-wave-over-fiber system providing up to $27 \mathrm{~Gb} / \mathrm{s}$ by using electrical 16QAM-OFDM IQ-modulation and optical up-conversion [7]. Here, bandwidth and spectral efficiency were $7 \mathrm{GHz}$ and $3.85 \mathrm{bit} / \mathrm{s} / \mathrm{Hz}$. A similar system using optical IQ-modulation was also reported by researchers from Chiao Tung University and Corning in 2010 [8]. A way to even higher capacities may be seen in using optical I/Q-modulation as well as optical coherent detection. As for systems operating at higher millimeter-wave systems, researchers at NTT have demonstrated $10.3125 \mathrm{~Gb} / \mathrm{s}$ and $8 \mathrm{~Gb} / \mathrm{s}$ at $125 \mathrm{GHz}$ [9]. At one point the world record was held by partner DTU [4]. In 2014, a group from Taiwan demonstrated $50 \mathrm{Gbit} / \mathrm{s}$ at $103 \mathrm{GHz}$ with $5 \mathrm{bit} / \mathrm{s} / \mathrm{Hz}$ spectral efficiency [10] and $84 \mathrm{Gbit} / \mathrm{s}$ in the $60 \mathrm{GHz}$ band [11], while a group from China, including ZTE, demonstrated 146Gbit/s in the Wband [12]. Most recent at OFC 2016, the UDE group demonstrate below FEC-limit $($ BER $<10-3)$ ultra-high spectral efficient $9 \mathrm{bit} / \mathrm{s} / \mathrm{Hz}$ wireless $60 \mathrm{GHz}$ transmission using a 512-QAM-OFDM modulation format and high throughput $21 \mathrm{~Gb} / \mathrm{s}$ wireless $60 \mathrm{GHz}$ transmission with a $6 \mathrm{bit} / \mathrm{s} / \mathrm{Hz}$ spectral efficiency [13]. The research conducted within FIWIN5G looks to go beyond these results and improve on these lab-based experiments by developing components and sub-systems to enable integrated and deployable systems. It sets as its goal to demonstrate world-record performance in optically supported wireless links. To do this, the research in this network aims to provide a number of key components and system developments as highlighted below.

\subsection{Millimetre-wave generation techniques}

There are multiple optical signal generation techniques, the most promising of which is optical heterodyning. This method requires an optical signal source generating two different wavelengths that are mixed into a photodiode or photoconductor (used as photo-mixer). The generated signal is an electrical beat-note at a frequency given by the difference between the wavelengths, presenting an unrivalled frequency tuning range from gigahertz to terahertz. However, when the two wavelengths are generated from uncorrelated sources, the 
generated beat-note exhibits large phase noise fluctuations due to the linewidth of the lasers and to the relative wavelength fluctuation between them [16]. This prevents the use of complex codes, with negative impact on the maximum data rate.

One recent approach used with heterodyne sources is to push the photonic integration, with the monolithic integration of two Distributed Feedback (DFB) lasers and light combining elements. Among the advantages brought by monolithic integration we can highlight the resulting compactness of the system and that the lasers will encounter the same environment fluctuations, thus reducing noise. The most common approach is to grow the two DFB lasers side by side. Using quantum-dash long cavities and combining the wavelengths using a Yjunction, tuning ranges from 2 to $20 \mathrm{GHz}$ and optical linewidth around $1 \mathrm{MHz}$ has been demonstrated [17]. A number of complementary approaches will be adopted in FIWIN5G with a specific focus on enable devices that can be monolithically integrated to enable low-cost and high efficiency devices. For example, one project will investigate millimetre-wave frequency generation using optical heterodyning of DFB lasers co-integrated on glass substrate.

\subsection{Photodiode Technologies}

In photodiode technology, devices with a $3 \mathrm{~dB}$ bandwidth in excess of $100 \mathrm{GHz}$ are available from Finisar, a partner in FIWIN5G (formerly $\mathrm{U}^{2} \mathrm{~T}$ ). High frequency and high output power PDs are one of the key elements in today's optical RoF links with high performance PDs actively investigated within the project [18]. Key objectives of the current research in high performance PD development is to enhance the operational frequency limitations and simultaneously to achieve high radio frequency (RF) power saturation levels without affecting the PD bandwidth. High-frequency operation requires an appropriate PD layer structure design, which comprises relatively thin absorption layers to reduce transit time limitations but thus handicaps the overall responsivity especially for top-illuminated PDs. In return, the resistance-capacitance (RC) limitations overweight due to the thin absorption layers requiring a dedicated layer structure design for balancing those limitations. Actually, the clear trend to obtain a high responsivity, a high bandwidth (or operational frequency for narrow-band applications) and a high output RF power is to use waveguide-type PDs rather than top-illuminated ones [19] as waveguide PDs provide more freedom in the designs of the PD layer structure and topology.

Recently, partners have reported a novel $1.55 \mu \mathrm{m} \operatorname{InGaAs}(\mathrm{P}) / \mathrm{InP}$ waveguide PDs utilizing a so-called triple transit region (TTR) layer structure which provide high output power levels in the mmW range. This enabled an experimental demonstration of a TTR-PDs exhibiting a $3 \mathrm{~dB}$ bandwidth beyond $110 \mathrm{GHz}$ and an output power level exceeding $0 \mathrm{dBm}(1 \mathrm{~mW})$ to be reported [20].

\section{TRAINING FUTURE RESEARCH LEADERS}

The technical focus of this project is at the intersection of a number of emerging technology areas. It requires its researchers to be well versed in a range of areas potentially covering wireless communications, high-frequency RF design, optical communications and data networking. It is debatable if any single research institute has a full and deep coverage of such topics in Europe. In addition, it is clear that mm-wave radio technologies are likely to have a huge impact in the market over the next decade as bandwidth demands increase. We argue that to enable the translation of academic research to industrial application there is a need for close collaboration and to provide early stage researchers with a comprehensive understanding of the challenges that industry face. We believe that this exposure, across disciplinary and sectoral groups, will offer a vital opportunity to advance researchers' understanding and lead to graduates highly skilled in the key technologies as well as in the key transferable skills needed to provide European leadership in this area for the future.

\section{CONCLUSIONS}

The FiWiN5G project has the dual aims of providing technological advances in combination with the training of a highly skilled researcher base in photonic technologies to support future $5 \mathrm{G}$ networks. It is aiming at the generation of devices and system demonstrations targeting 100Gibt/s and 400Gbit/s wireless links, technologies to enable transmission links in the $180 \mathrm{GHz}$ band, optimised modulation schemes for high bandwidth communications, steerable antennas, as well as optical sampling technologies. These techniques have been identified as clear requirements in future networks where photonics techniques have advantageous properties. To support these techniques a number of device technologies have been identified including frequency generation using optical heterodyning of DFB Lasers co-integrated on glass substrates, photonic analogue to digital converters, high-bandwidth and high-power photodiodes and monolithically integrated photodiodes and antennas.

\section{ACKNOWLEDGEMENTS}


This project has received funding from the European Union's Horizon 2020 research and innovation programme 2014-2018 under grant agreement No. 642355. The author would like to thank all partners and partner institutions for their input and efforts in the programme.

\section{REFERENCES}

[1] M. Weiß, M. Huchard, A. Stöhr, B. Charbonnier, "60GHz Photonic Millimeter-Wave Link for Short to Medium Range Wireless Transmission up to $12.5 \mathrm{~Gb} / \mathrm{s}$,“ IEEE J. Ligthwave Technol., vol. 26, no. 15, August 2008, pp. 2424-2429

[2] http://www.ist-iphobac.org/ng/ Project reference: 619870

[3] M. Weiß, et al., "27 Gb/s Photonic Wireless $60 \mathrm{GHz}$ Transmission System using 16-QAM OFDM“, IEEE Int. Microwave Photonics Conference, Valencia, October 2009, (post-deadline)

[4] X. Pang et al., "100 Gb/s hybrid optical fiber-wireless link in the W-band (75-110 GHz)", Optics Express, vol. 19 , no. 25,5 December 2011

[5] G. Chang et al., "Architectures and Technologies for very High-Throughput In-Building Wireless Services using Radio-over-Fiber Networks", IEEE LEOS 2009, pp. 37-38, 2009

[6] A. Stöhr et al., "60 GHz Radio-over-Fiber Technologies for Broadband Wireless Services”, OSA Journal of Optical Networking, 8(5), May 2009

[7] A. Stöhr et al., "Millimeter-Wave Photonic Components for Broadband Wireless Systems", IEEE Microwave Theory and Techniques, vol.58, no.11, pp.3071-3082, 2010

[8] C.-T. Liu et al., "21 Gbps OFDM-8QAM Radio-over-Fiber System with $10 \mathrm{~m}$ Wireless Transmission at 60 GHz Employing a Simple System Architecture, OFC/NFOEC 2010, San Diego, paper OTuF4

[9] A. Hirata et al., "120-GHz-Band Millimeter-Range Photonic Wireless Link for 10-Gb/s Data Transmission", IEEE Trans. Microwave Theory and Techniques, vol. 54, no. 5, pp. 1937-1943, May 2006

[10] C-H Ho et al. "5-bit/s/Hz 50-Gbps W-band Optical/ Wireless System Employing Single- Sideband SingleCarrier Modulation" OFC2014 M2D.2

[11] C-T Lin et al. "84-Gbps 64-QAM 2×2 MIMO RoF System at 60 GHz Employing Single- Sideband SingleCarrier Modulation" OFC2014 M3D.1

[12] X. Li, et al. "Antenna Polarization Diversity for 146Gb/s Polarization Multiplexing QPSK Wireless Signal Delivery at W-band" OFC2014 M3D.7

[13] A. Stöhr, B. Shih, S. Abraha, A. Steffan, and A. Ng'oma, "High Spectral-Efficient 512-QAM-OFDM 60 GHz CRoF System using a Coherent Photonic Mixer (CPX) and an RF Envelope Detector," in Optical Fiber Communication Conference, paper Tu3B.4, 2016

[14] “Cisco Visual Networking Index: Global Mobile Data Traffic Forecast Update, 2012-2017," Cisco Corp., Feb. 2013.

[15] O. Omomukuyo, M.P. Thakur, J.E Mitchell "A Simple 60-GHz MB-OFDM Ultra-Wideband RoF System Based on Remote Heterodyning”, IEEE Photon. Technol. Letts 25(3): pp. 268-271, Feb. 2013

[16] F. Van-Dijk, A. Accard, A. Enard, O. Drisse, D. Make, F. Lelarge, "Monolithic dual wavelength DFB lasers for narrow linewidth heterodyne beat-note generation," International Topical Meeting on Microwave Photonics 2011, pp.73-76, 18-21 Oct. 2011

[17] C. C. Renaud, "Ultra-high-speed uni-traveling carrier photodiodes and their applications," OFC 2013, 1-3 (2013).

[18] E. Rouvalis, M. Chtioui, M. Tran, F. Lelarge, F. Van Dijk, M. J. Fice, C. C. Renaud, G. Carpintero, and A. J. Seeds, "High-speed photodiodes for InP-based photonic integrated circuits," Opt. Express 20, 9172-9177 (2012).

[19] V. Rymanov, A. Stöhr,1, S. Dülme, and T. Tekin, “Triple transit region photodiodes (TTR-PDs) providing high millimeter wave output power," Optics Express, 22 (7), Mar 2014 